\title{
PRESENCIA DE RHEIDAE EN TIERRA DEL FUEGO DURANTE LA TRANSICIÓN PLEISTOCENO-HOLOCENO. IMPLICANCIAS BIOGEOGRÁFICAS Y PALEOECOLÓGICAS.
}

FABIANA M. MARTIN", MAURICIO MASSONE*", ALFREDO PRIETO*** Y PEDRO CÁRDENAS"***

\section{RESUMEN}

En este trabajo presentamos de manera preliminar información sobre una fecha taxón de 9.960 \pm 50 años AP, obtenida para una falange primera de Rheidae procedente del sitio Tres Arroyos 1, norte de Tierra del Fuego, Chile. Se discuten sus implicancias biogeográficas, paleoecológicas y algunas consideraciones arqueológicas.

PALABRAS CLAVES: Rheida, Pleistoceno, Tierra del Fuego, Fecha Taxon.

\section{RHEIDAE PRESENCE DURING PLEISTOCENE-HOLOCENE TRANSITION IN TIERRA DEL FUEGO. BIOGEOGRAPHICAL AND PALAEOECOLOGICAL IMPLICATIONS.}

\section{ABSTRACT}

Preliminary information related with a taxon-date of $9.960 \pm 50$ years BP for Rheidae will be introduced in this paper. This date was obtained on a Rheidae first phalanx from the Tres Arroyos 1 site, north of Tierra del Fuego, Chile. Biogeographical, palaeoecological and archaeological implications are discussed.

KEY WORDS: Rheidae, Pleistocene, Tierra del Fuego, Taxon Date.

\section{INTRODUCCIÓN}

Una evaluación y planteo biogeográfico acerca de la arqueología de la parte meridional de América del Sur (Borrero 1989-1990), indicó la expectativa de evolución divergente para poblaciones humanas que pudieron haber quedado aisladas tras la formación del estrecho de Magallanes. Entre las razones para esperar dicha divergencia consideró, además de la restricción implicada en un ambiente insular,

* Fundación CEQUA. Av. Bulnes 01890, Punta Arenas, Chile. Email: fabiana.martin@cequa.cl

* Museo Natural de Concepción, DIBAM, Maipú 2356, Concepción, Chile. Email: mmassone@surnet.cl

** Centro de Estudios del Hombre Austral, UMAG. Av. Bulnes 01890, Punta Arenas, Chile. Email: alfredo.prieto@umag.cl

*e* Centro de Estudios del Hombre Austral, UMAG. Av. Bulnes 01890, Punta Arenas, Chile. Email: alfredo.prieto@umag.cl 
la necesidad de los primeros cazadores de acudir a novedosas estrategias y tácticas de supervivencia. Esto último se basaba, entre otras cosas, en la ausencia en la isla de ciertas presas potenciales como el huemul (Hippocamelus bisulcus) y el ñandú (Rhea americana, Pterocnemia pennata) [Texera 1973]. En este trabajo informamos sobre el primer registro de ñandú en un contexto de la Transición Pleistoceno-Holoceno de la isla, recuperado en el sitio arqueológico Tres Arroyos 1, Tierra del Fuego, Chile.

\section{IMPORTANCIA DE RHEIDAE}

No son abundantes las pruebas de consumo de Rheidae en los sitios arqueológicos de la Patagonia continental. De hecho, la mayoría de los hallazgos corresponden a cáscaras de huevo atribuidas a Rheidae (Caviglia y Figuerero Torres 1976: 318; Gómez Otero 1989-90; Crivelli et al. 1996: 208, 210 , entre otros), o a Rhea americana o Pterocnemia pennata en el norte de Patagonia (Tonni 1981; Menegaz 1996). Muchas veces estas cáscaras aparecen quemadas (Menegaz 1996: 148). Aunque también se han identificado sus restos óseos (por ejemplo Salemme y Miotti 1998; Mena y Jackson 1991; Miotti 1998).

La profundidad temporal de los hallazgos de Rheidae raramente es alta. Las primeras evidencias se registraron en los depósitos más antiguos de los sitios Piedra Museo (Miotti 1998), Los Toldos 3 (Miotti 1998) y Casa del Minero 1 (Paunero et al. 2004). Hay que destacar que en Piedra Museo están presentes las dos especies (Miotti 1996), que hoy son alopátricas. A nivel de la región de Magallanes, sabemos que se encuentra representado en los niveles inferiores de cueva Fell (Saxon 1979). Sin embargo, las únicas fechas taxón pleistocenas disponibles proceden de cueva de los Chingues, Pali-Aike (Martin 2008).

De todas maneras, aunque los restos recuperados no sean abundantes, muchos de los huesos de ñandú registrados tienen huellas de corte (Crivelli Montero et al. 1996; Menegaz 1996:148; Miotti 1998; Fernández 2000: 576-7) y, en los escasos estudios detallados, también marcas de percusión. Esto apoya la idea de consumo intensivo de los mismos. No entraremos en la discusión tafonómica acerca de la visibilidad de restos de Rheidae en sitios arqueológicos (ver Belardi 1999; Fernández, 2000; Cruz y Elkin 2003; Fernández et al. 2001), pero es claro que estos animales tenían un lugar en las dietas prehistóricas.

\section{EL HALLAZGO}

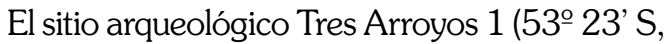
68ํㅜㄱ' O) está emplazado en el cerro de los Onas, un afloramiento rocoso formado por sedimentos terciarios que conforman la sierra Carmen Silva en el norte de Tierra del Fuego. El sitio fue descubierto en la década del 1960 por uno de sus ex propietarios, el señor Carlos Descourvieres. Con posterioridad, fue visitado por Annette Laming-Emperaire y en 1975 por Earl Saxon, quien excavó un pequeño sondeo recuperando restos culturales que fechó en $135 \pm 85$ AP [MC-1070] (Rapaire y Hugues 1977; Saxon 1979). Sin embargo, fueron los trabajos de Mauricio Massone, a lo largo de dos décadas, los que mostraron el potencial y la importancia del sitio (Massone 1987; Massone et al. 1993; Massone 2004). Durante ese período se excavaron seis unidades que fueron denominadas A, B, Contacto A/B, C, D y $E$ de tamaños y profundidades variables. En líneas generales se reconocieron estratigráficamente seis niveles que fueron numerados sucesivamente del I (nivel superior) al VI (nivel inferior). Los Niveles I a IV están asociados a ocupaciones humanas del Holoceno Tardío, mientras que el Nivel V presentó evidencias de asociación humana con fauna del Pleistoceno (Massone 1987, 2004).

Esta secuencia estratigráfica está asociada a una secuencia cronológica que abarca más de 12.000 años, con fechas que incluyen desde edades "modernas" a máximas de $12.540 \pm 70$ [Beta-123152] (Rapaire y Hugues 1977; Massone 1987; Borrero 2003; Massone 2004).

Los trabajos arqueológicos a lo largo de dos décadas mostraron abundantes restos de mamiferos terrestres, que incluían los taxa de edad pleistocena más comunes hallados en el continente. Los mismos fueron inicialmente descriptos por Caviglia (19851986), Mengoni Goñalons (1987) y Massone (1987). Posteriormente la lista de especies fue ampliándose (Prieto y Canto 1997; Latorre 1998; Martin y Borrero 1999; Alberdi y Prieto 2000; Prevosti 2006, Weinstock et al. 2009). En este trabajo presentamos una nueva evidencia que consiste en dos especímenes 
óseos de Rheidae ${ }^{1}$. Se trata de una falange primera proximal y una falange segunda completa. Ambas se encuentran muy bien preservadas y registran marcas de roedores. La falange primera, cuyo número de inventario es el 9410, procede de la cuadrícula C, Nivel IV (cuya profundidad oscila entre los 45-60 a $70 \mathrm{~cm}$ ) y fue fechada en $9960 \pm 50 \mathrm{AP}(\mathrm{GX}-32667)$, $\delta^{13} \mathrm{C}-21.1 \% 0, \delta^{15} \mathrm{~N}+4.9 \%$. La falange segunda procede de la unidad Contacto A-B, Nivel III.

\section{DISCUSIÓN Y CONCLUSIONES}

Respecto a la ubicación estratigráfica de los hallazgos queremos destacar que el sitio Tres Arroyos presenta evidencias de migración vertical de restos óseos y artefactos líticos (Jackson, 2002; Borrero, 2003; Massone y Prieto, 2004). La principal razón la constituye la acción de conejo europeo, cuyos restos, introducidos a la isla exitosamente en la década de 1930 (Goodall, 1975), aparecen en los depósitos correspondientes al Pleistoceno tardío (Martin y Borrero 1999). El ejemplo más conocido es la mandíbula de Dusicyon avus recuperada en el Nivel III, fechada en $10.575 \pm 65$ AP, Oxa-9245 (Massone y Prieto 2004). Por eso es que se ha adoptado la necesidad de defender las edades de distintos taxa a través de fechados directos (Borrero 1997). Podemos respaldar que uno de los ejemplares aquí presentado corresponde a tiempos de la Transición Pleistoceno/Holoceno sobre la base de una fecha taxón. Esto, a su vez, llevará a reevaluaciones de las estrategias de subsistencia prevalecientes en los tiempos de las primeras ocupaciones humanas de Tres Arroyos 1 y a nuevas comparaciones con los casos registrados en el continente.

El hallazgo de Rheidae en un contexto arqueológico de la Isla Grande de Tierra del Fuego es una contribución a la biogeografía de estas aves no voladoras. Esta evidencia no sólo constituye otra prueba independiente de la antigua conexión de Tierra del Fuego al continente (Clapperton 1992), sino que también nos indica que debemos ampliar la distribución hacia el sur de Rheidae, conocida previamente para la transición Pleistoceno-Holoceno. Los procesos que modificaron esa distribución y los que llevaron a la desaparición de ese taxón

1 Ambos especímenes se encuentran almacenados en el Centro de Estudios del Hombre Austral, Instituto de la Patagonia, Universidad de Magallanes, Punta Arenas, Chile. en la isla, aún deben ser evaluados, pero sin dudas implican cambios en la dinámica y paleoecología de los ambientes habitados por cazadores humanos en Fuego-Patagonia a fines del Pleistoceno. Por otra parte, mas especificamente, amplía nuestra comprensión del conjunto faunístico asociado con las primeras ocupaciones humanas de Tierra del Fuego. En relación con el tema de la evolución divergente, tal como lo planteara Borrero (1989-1990), entendemos que dada la edad de esta presencia, no se contrasta negativamente la hipótesis de evolución divergente, la que debe juzgarse sobre la base de desarrollos culturales posteriores en el tiempo. Sólo se plantean sobre una base más completa las condiciones iniciales de la relación entre poblaciones humanas y recursos alimenticios en tiempos de las primeras ocupaciones registradas en la actual isla Grande de Tierra del Fuego.

\section{AGRADECIMIENTOS}

Queremos expresar nuestro agradecimiento a Jaime Cárcamo del laborarorio de zoología, Instituto de la Patagonia, Umag, por facilitarnos materiales de referencia. A Luis A. Borrero por la lectura crítica del manuscrito. Finalmente, los restos óseos referidos en la nota fueron recuperados en el marco del proyecto FONDECYT № 1960027 , Hombre temprano y paleoambiente en Tierra del Fuego, dirigido por Mauricio Massone.

\section{BIBLIOGRAFÍA}

ALBERDI, M. T. y A. PRIETO, 2000. Hippidion (Mammalia, Perissodactyla) de las cuevas de de las Provincias de Magallanes y Tierra del Fuego. Anales del Instituto de la Patagonia (Serie Ciencias Sociales) 28:147-171.

BELARDI, J.B., 1999. Hay choiques en la terraza. Información tafonómica y primeras implicaciones arqueofaunísticas para Patagonia. Arqueología 9: 163-185.

BORRERO, L. A., 1997. La extinción de la megafauna en la Patagonia. Anales del Instituto de la Patagonia (Serie Ciencias Sociales) 25:89-102.

BORRERO, L. A., 1989-1990. Evolución cultural divergente en la Patagonia Austral. Anales del Instituto d ela Patagonia (Serie Ciencias Sociales) 19: 133-140.

BORRERO, L. A., 2003. Taphonomy of the Tres Arroyos 1 rockshelter, Tierra del Fuego, Chile. Quaternary International 109-110:87-93. 
CAVIGLIA, S. E., 1985-1986. Restos de cánidos tempranos en Fuego-Patagonia. Anales del Instituto de la Patagonia (Serie Ciencias Sociales) 16:85-93.

CAVIGLIA, S. E. y M. J. FIGUERERO TORRES, 1976. Material faunístico de Las Buitreras. Relaciones 10: 315-319.

CLAPPERTON, C. M., 1992. La última glaciación y deglaciación en el estrecho de Magallanes: implicaciones para el poblamiento en Tierra del Fuego. Anales del Instituto de la Patagonia 21:113-128.

CRIVELLI, E. A., U. PARDIÑAS, M. M. FERNÁNDEZ, M. BOGAZZI, A. CHAUVIN, V. FERNÁNDEZ y M. LEZCANO, 1996. Cueva Epullán Grande (Pcia. del Neuquen). Informe de avance. Praehistoria 2: 185-265.

CRUZ, I. y D. ELKIN, 2003. Bone Structural Density of the Lesser Rhea (Pterocnemia pennata) (Aves: Rheidae). Taphonomic and Archaeological Implications. Journal of Archaeological Science 30: 37-44.

FERNÁNDEZ, P., 2000. Rendido a tus pies: acerca de la composición anatómica de los conjuntos arqueofaunísticos con restos de Rheiformes de Pampa y Patagonia. Desde el País de los Gigantes. Perspectivas arqueológicas en Patagonia, II: 573-586, Universidad Nacional de la Patagonia Austral, Río Gallegos.

FERNÁNDEZ, P., I. CRUZ y D. ELKIN, 2001. Densidad mineral ósea de Pterocnemia pennata (Aves: Rheidae). Una herramienta para evaluar frecuencias anatómicas en sitios arqueológicos. Relaciones de la Sociedad Argentina de Antropología 26: 243-260.

GÓMEZ OTERO, J., 1989-1990. Cazadores tardíos en la zona fronteriza del paralelo 52 Sur. I. El paraje de Juni Aike. Anales del Instituto de la Patagonia 19: 47-71.

GOODALL, R. N. P, 1975. Tierra del Fuego. Ediciones Shanamiim, Buenos Aires-Ushuaia.

JACKSON, D. 2002. Los instrumentos líticos de los primeros cazadores de Tierra del Fuego. Santiago: Dirección de Bibliotecas, Archivos y Museos.

LATORRE, C. 1998. Paleontología de mamíferos del Alero Tres Arroyos 1, Tierra del Fuego, XII Region, Chile. Chile. Anales del Instituto de la Patagonia (Serie Ciencias Naturales) 26:77-90.

MARTIN, F. M. y L. A. BORRERO, 1999. Los pequeños mamíferos de Tres Arroyos 1, Tierra del Fuego, Chile. El caso de los conejos, en Informe final, Proyecto FONDECYT Número 1960027. Hombre Temprano y paleoambiente en Tierra del Fuego. MS.

MARTIN, F. M., 2008. Bone Crunching Felids at the End of the Pleistocene in Fuego-Patagonia, Chile. Journal of Taphonomy 6(3-4): 337-372.
MASSONE, M., 1987. Los cazadores paleoindios de Tres Arroyos (Tierra del Fuego). Anales del Instituto de la Patagonia (Serie Ciencias Sociales) 47-60.

MASSONE, M., 2004. Los cazadores después del hielo. Santiago: Ediciones de la Dirección de Bibliotecas Archivos y Museos.

MASSONE, M. y A. PRIETO, 2004. Evaluación de la modalidad cultural Fell 1 en Magallanes. Chungara Volumen especial, pp. 303-315.

MASSONE, M., D. JACKSON y A. PRIETO, 1993. Perspectiva Arqueológica de los Selk'nam. Santiago de Chile: Centro de Investigaciones Diego Barros Arana, Dirección de Bibliotecas, Archivos y Museos.

MENA, F. y D. JACKSON, 1991. Tecnología y subsistencia en Alero Entrada Baker. Anales del Instituto de la Patagonia 20: 169-203.

MENEGAZ, A., 1996. Análisis del material faunístico procedente del sitio Piedra del Aguila 11 (Neuquen, Argentina) Praehistoria 2: 147-171.

MENGONI GOÑALONS, G. L., 1987. Modificaciones culturales y animales en los huesos de los niveles inferiores del sitio Tres Arroyos 1 (Tierra del Fuego, Chile). Anales del Instituto de la Patagonia (Serie Ciencias Sociales) 17:61-66.

MIOTTI, L. (1996). Piedra Museo (Santa Cruz): Nuevos datos para la ocupacion Pleistocenica en Patagonia. (Ed. J. Gomez Otero), Arqueologia, Solo Patagonia, CENPATCONICET, Puerto Madryn, pp. 27-38.

MIOTTI, L., 1998. Zooarqueología de la meseta central y costa de Santa Cruz. Museo de Historia Natural, San Rafael.

PAUNERO, R. S., M. CUETO, A. FRANK, G. GHIDINI, G. ROSALES y F. SKARBUN, 2004. Comunicación sobre campaña arqueológica 2002 en localidad La María, Santa Cruz. Contra Viento y Marea. Arqueología de Patagonia (Eds. M. T. Civalero, P. M. Fernández, y A. G. Guráieb), pp. 797-808. INAPL-SAA, Buenos Aires.

PREVOSTI, F. J., 2006. New material of Pleistocene cats (Carnivora Felidae) from Southern South America with comments on biogeography and the fossil record. Geobios 39:649-679.

PRIETO, A. y J. CANTO, 1997. Presencia de un Lamoide atípico en la cueva Lago Sofía 4 (Ultima Esperanza) y Tres Arroyos (Tierra del Fuego) Región de Magallanes, Chile. Anales del Instituto de la Patagonia (Serie Ciencias Sociales) 25:147- 150.

RAPAIRE, J. L. y G. HUGUES. 1977. Monaco radiocarbon mesurements V. Radiocarbon 19 (1):49-61. 
SALEMME, M. y L. MIOTTI, 1998. Ths Status of Rheids in Patagonia: Environmental Approach and Economic Interpretation during the Late Pleistocene/Early Holocene Transition. Poster presentado al 8o. International Congress of Archaeozoology, Victoria.

SAXON, E. C. 1979. Natural Prehistory: The Archaeology of Fuego-Patagonian Ecology. Quaternaria XXI:329-356. I Instituto de la Patagonia (Serie Ciencias Naturales) 26:77-90.

TEXERA, W. A., 1973. Distribución y diversidad de mamíferos y aves en la Provincia de Magallanes. III. Historia geológica de los géneros de mamíferos nativos terrestres.
Anales del Instituto de la Patagonia IV (1y3):307-320, Punta Arenas.

TONNI, E. P., 1981. Restos de aves hallados en el Alero de los Sauces. Trabajos de Prehistoria 1: 89-92.

WEINSTOCK, J., B. SHAPIRO, A. PRIETO, J. C. MARÍN, B. A. GONZÁLEZ, P. GILBERT M. THOMAS, E. WILLERSLEV. The Late Pleistocene distribution of vicuñas (Vicugna vicugna) and the "extinction"of the gracile llama ("Lama gracilis"): New molecular data. Quaternary Science Reviews (2009), doi:10.1016/ j.quascirev.2009.03.008. 
